\title{
ON TWO CLASSES OF REGULAR SEQUENCES
}

\author{
RI-XIANG CHEN
}

\begin{abstract}
In this paper we get two new classes of regular sequences in the polynomial ring over the field of complex numbers.
\end{abstract}

1. Introduction. Throughout this paper, $S=k\left[x_{1}, x_{2}, \ldots, x_{n}\right]$ denotes the polynomial ring in $n$ variables over a field $k$. Let $f_{1}, f_{2}, \ldots, f_{n}$ be some homogeneous polynomials in $S$ of degrees $a_{1}, a_{2}, \ldots, a_{n}$ with $1 \leq a_{1} \leq a_{2} \leq \cdots \leq a_{n}$. It is well known that the following statements are equivalent:

(1) $f_{1}, f_{2}, \ldots, f_{n}$ is a regular sequence in $S$;

(2) The Koszul complex $K .\left(f_{1}, f_{2}, \ldots, f_{n}\right)$ is the minimal free resolution of $S /\left(f_{1}, f_{2}, \ldots, f_{n}\right)$ over $S$

(3) $\left(f_{1}, f_{2}, \ldots, f_{n}\right)_{N}=S_{N}$ where $N=1+\sum_{i=1}^{n}\left(a_{i}-1\right)$;

(4) $\sqrt{\left(f_{1}, f_{2}, \ldots, f_{n}\right)}=\left(x_{1}, x_{2}, \ldots, x_{n}\right)$, that is, ht $\left(f_{1}, f_{2}, \ldots, f_{n}\right)=n$;

and when the field $k$ is algebraically closed, they are all equivalent to the following:

(5) $V\left(f_{1}, f_{2}, \ldots, f_{n}\right)=\{(0,0, \ldots, 0)\}$, that is, $(0,0, \ldots, 0)$ is the only solution to the system of equations $f_{1}=0, f_{2}=0, \ldots, f_{n}=0$.

There are some other criterions for $f_{1}, f_{2}, \ldots, f_{n}$ being a regular sequence. Despite the many criterions, there remain some very difficult questions about regular sequences.

For example, in [2], Conca, et al., made the following conjecture:

Conjecture 1.1. [2]. In $S=\mathbb{C}\left[x_{1}, x_{2}, x_{3}\right]$, let $p_{a}(3)=x_{1}^{a}+x_{2}^{a}+x_{3}^{a}$, $p_{b}(3)=x_{1}^{b}+x_{2}^{b}+x_{3}^{b}$ and $p_{c}(3)=x_{1}^{c}+x_{2}^{c}+x_{3}^{c}$, where $a, b, c \in \mathbb{N}^{*}$,

2010 AMS Mathematics subject classification. Primary 05E05, 13A02, Secondary 15A03, 54A10.

Keywords and phrases. Regular sequence, power sum, matrix, distance.

This work was partially supported by the National Natural Science Foundation of China (grant No. 11326064).

Received by the editors on January 5, 2015. 
$a<b<c, \operatorname{gcd}(a, b, c)=1$ and $6 \mid a b c$, then $p_{a}(3), p_{b}(3)$ and $p_{c}(3)$ form a regular sequence in $S$.

Conca, et al., verified some special cases of this conjecture, and the conjecture remains mysterious. Another conjecture about regular sequences is the famous EGH conjecture, which is wide open except some special cases.

Conjecture 1.2. [4]. If $I \subset S=k\left[x_{1}, x_{2}, \ldots, x_{n}\right]$ is a homogeneous ideal containing a regular sequence of homogeneous polynomials $f_{1}, f_{2}, \ldots, f_{n}$ of degrees $2 \leq a_{1} \leq a_{2} \leq \cdots \leq a_{n}$, then there exists a homogeneous ideal in $S$ containing $x_{1}^{a_{1}}, x_{2}^{a_{2}}, \ldots, x_{n}^{a_{n}}$ with the same Hilbert function.

These conjectures motivate us to further our study of regular sequences. It is well known that, generically, $f_{1}, f_{2}, \ldots, f_{n}$ form a regular sequence in $S$ (see Proposition 3.1), but we do not have many specific examples of regular sequences. The most popular example is the sequence $x_{1}^{a_{1}}, x_{2}^{a_{2}}, \ldots, x_{n}^{a_{n}}$. Also, if under a monomial order $<, \operatorname{in}_{<}\left(f_{1}\right)=$ $x_{1}^{a_{1}}, \operatorname{in}_{<}\left(f_{2}\right)=x_{2}^{a_{2}}, \ldots, \operatorname{in}_{<}\left(f_{n}\right)=x_{n}^{a_{n}}$, then $f_{1}, f_{2}, \ldots, f_{n}$ form a regular sequence in $S$. In addition, in the case of $a_{1}=a_{2}=\cdots=a_{n}=1$, $f_{1}, f_{2}, \ldots, f_{n}$ is a regular sequence if and only if $f_{1}, f_{2}, \ldots, f_{n}$ are $k$ linearly independent, and, equivalently, the determinant of the $n \times n$ coefficient matrix is nonzero.

It is easy to see that, if $f_{1}=g_{1} h_{1}$ with $\operatorname{deg}\left(g_{1}\right) \geq 1$ and $\operatorname{deg}\left(h_{1}\right) \geq 1$, then $f_{1}, f_{2}, \ldots, f_{n}$ is a regular sequence if and only if both $g_{1}, f_{2}, \ldots, f_{n}$ and $h_{1}, f_{2}, \ldots, f_{n}$ are regular sequences. So, in [1], Abedelfatah considered the following class of regular sequences. Let $f_{1}, f_{2}, \ldots, f_{n}$ be homogeneous polynomials which split into linear factors, then $f_{1}, f_{2}, \ldots, f_{n}$ is a regular sequence if and only if for $i=1,2, \ldots, n$ and for every linear factor $l_{i}$ of $f_{i}, l_{1}, l_{2}, \ldots, l_{n}$ is a regular sequence. Abedelfatah proved that the EGH conjecture holds for this class of regular sequences.

The goal of this paper is to get two new classes of regular sequences in the polynomial ring over the field of complex numbers.

In Section 2, we consider the power sum symmetric polynomial $p_{m}(n)=x_{1}^{m}+x_{2}^{m}+\cdots+x_{n}^{m}$ in $S=\mathbb{C}\left[x_{1}, x_{2}, \ldots, x_{n}\right]$. We prove that, if $a, d \in \mathbb{N}^{*}$ with $\operatorname{gcd}(a, d)=1$ and $n ! \mid a(a+d) \cdots(a+(n-1) d)$, 
then $p_{a}(n), p_{a+d}(n), \ldots, p_{a+(n-1) d}(n)$ is a regular sequence in $S$ (see Theorem 2.4.)

In Section 3, we prove that, if $f_{1}, f_{2}, \ldots, f_{n}$ are homogeneous polynomials of degrees $a_{1}, a_{2}, \ldots, a_{n}$ in $S=\mathbb{C}\left[x_{1}, x_{2}, \ldots, x_{n}\right]$ and for all $i=1,2, \ldots, n$, the distance between $f_{i}$ and $x_{i}^{a_{i}}$ (see Definition 3.4) is less than 1 , then $f_{1}, f_{2}, \ldots, f_{n}$ is a regular sequence in $S$ (see Theorem 3.5.)

2. Regular sequences of power sums. Throughout this section, $S=\mathbb{C}\left[x_{1}, x_{2}, \ldots, x_{n}\right]$, and let $p_{m}(n)$ denote the power sum symmetric polynomial $x_{1}^{m}+x_{2}^{m}+\cdots+x_{n}^{m}$ in $S$. Given positive integers $a_{1}<$ $a_{2}<\cdots<a_{n}$, let $A$ be the set $\left\{a_{1}, a_{2}, \ldots, a_{n}\right\}$. For simplicity, we will denote the sequence $p_{a_{1}}(n), p_{a_{2}}(n), \ldots, p_{a_{n}}(n)$ in $S$ by $p_{A}(n)$.

The question is: when is $p_{A}(n)$ a regular sequence in $S$ ? To answer it, Conca, et al., made the following two observations.

Lemma 2.1. [2]. Let $a_{1}<a_{2}<\cdots<a_{n}$ be positive integers and $A=$ $\left\{a_{1}, a_{2}, \ldots, a_{n}\right\}$. Set $d=\operatorname{gcd}\left(a_{1}, a_{2}, \ldots, a_{n}\right)$ and $A^{\prime}=\{a / d \mid a \in A\}$. Then $p_{A}(n)$ is a regular sequence in $S$ if and only if $p_{A^{\prime}}(n)$ is a regular sequence in $S$.

Lemma 2.2. [2]. Let $f_{1}, f_{2}, \ldots, f_{n}$ be a regular sequence of homogeneous symmetric polynomials of degrees $1 \leq a_{1} \leq a_{2} \leq \cdots \leq a_{n}$ in $S=$ $\mathbb{C}\left[x_{1}, x_{2}, \ldots, x_{n}\right]$. Then $n$ ! divides $a_{1} a_{2} \cdots a_{n}$, that is, $n ! \mid a_{1} a_{2} \cdots a_{n}$.

By Lemma 2.1, we can always assume that $\operatorname{gcd}\left(a_{1}, a_{2}, \ldots, a_{n}\right)=1$. And Lemma 2.2 implies that $n ! \mid a_{1} a_{2} \cdots a_{n}$ is a necessary condition for $p_{A}(n)$ to be a regular sequence in $S$. One may wonder if both $\operatorname{gcd}\left(a_{1}, a_{2}, \ldots, a_{n}\right)=1$ and $n ! \mid a_{1} a_{2} \cdots a_{n}$ would imply $p_{A}(n)$ being a regular sequence. This is true for $n=2$, which is proved in [2], and is also a special case of the following Theorem 2.4. The case of $n=3$ remains mysterious as Conjecture 1.1. However, for $n \geq 4$, the above two conditions are not sufficient for $p_{A}(n)$ being a regular sequence, as is illustrated in the following example.

Example 2.3. $\operatorname{gcd}(1,3,5,24)=1$ and $4 ! \mid 1 \times 3 \times 5 \times 24$, but $p_{1}(4), p_{3}(4), p_{5}(4), p_{24}(4)$ is not a regular sequence in $S=\mathbb{C}\left[x_{1}, x_{2}, x_{3}, x_{4}\right]$, because $\left(e^{\pi i / 24},-e^{\pi i / 24}, 1,-1\right)$ is a nonzero solution to the system of 
equations $p_{1}(4)=0, p_{3}(4)=0, p_{5}(4)=0, p_{24}(4)=0$. Examples can be similarly constructed for $n>4$.

In [2, Proposition 2.9], Conca, et al., proved that if $a_{1}, a_{2}, \ldots, a_{n}$ are consecutive positive integers, then $p_{A}(n)$ is a regular sequence in $S$. To generalize this result, we have the following theorem.

Theorem 2.4. Let $S=\mathbb{C}\left[x_{1}, x_{2}, \ldots, x_{n}\right], n \geq 2, a, d \in \mathbb{N}^{*}$ and $A=\{a, a+d, a+2 d, \ldots, a+(n-1) d\}$. Assume that $\operatorname{gcd}(a, d)=1$ and $n ! \mid a(a+d)(a+2 d) \cdots(a+(n-1) d)$. Then $p_{A}(n)$ is a regular sequence in $S$.

Note that the assumption $\operatorname{gcd}(a, d)=1$ is equivalent to $\operatorname{gcd}(a, a+$ $d, a+2 d, \ldots, a+(n-1) d)=1$. And, when $d=1$, this theorem is [2, Proposition 2.9]. To prove Theorem 2.4, we need to prove the following two lemmas, which themselves are interesting.

Lemma 2.5. Let $n, a, d \in \mathbb{N}^{*}, n \geq 2$ and $\operatorname{gcd}(a, d)=1$. Then $n ! \mid a(a+d)(a+2 d) \cdots(a+(n-1) d)$ if and only if $\operatorname{gcd}(d, n !)=1$, that is, either $d=1$ or every prime factor of $d$ is greater than $n$.

Proof. Only if. Suppose, for contradiction, that there is a prime number $p$ which divides $\operatorname{gcd}(d, n !)$. Then $p \mid d$ and $p \mid n$ !. Since $n$ ! | $a(a+d)(a+2 d) \cdots(a+(n-1) d)$, it follows that $p \mid a+i d$ for some $0 \leq i \leq n-1$, which implies that $p$ is a prime factor of $\operatorname{gcd}(d, a+i d)=\operatorname{gcd}(d, a)$. But $\operatorname{gcd}(a, d)=1$, so we get a contradiction and, therefore, $\operatorname{gcd}(d, n !)=1$.

If. Assume $\operatorname{gcd}(d, n !)=1$. Then there exist integers $s, t$ such that $s d+t(n !)=1$. Hence,

$$
\begin{aligned}
& s^{n} a(a+d)(a+2 d) \cdots(a+(n-1) d) \\
& \equiv s a(s a+s d)(s a+2 s d) \cdots(s a+(n-1) s d) \\
& \equiv s a(s a+1-t(n !))(s a+2-2 t(n !)) \cdots \\
& \quad(s a+(n-1)-(n-1) t(n !)) \\
& \equiv s a(s a+1)(s a+2) \cdots(s a+(n-1)) \\
& \equiv 0 \quad(\bmod n !) .
\end{aligned}
$$


Since $\operatorname{gcd}(s, n !)=1$, it follows that $n ! \mid a(a+d)(a+2 d) \cdots(a+$ $(n-1) d)$.

In [5], Lam and Leung proved that if $z_{1}, z_{2}, \ldots, z_{n} \in \mathbb{C}$ are some $d$ th roots of unity and $z_{1}+z_{2}+\cdots+z_{n}=0$, then $n$ must be a linear combination with non-negative integer coefficients of the prime factors of $d$. This is the main theorem of [5], and it was proved by group ring techniques. The next lemma can be viewed as a corollary to Lam and Leung's theorem. For the completeness of the paper, we also give an elementary proof of the lemma.

Lemma 2.6. Let $d, n \in \mathbb{N}^{*}, d \geq 2, n \geq 2$ and $z_{1}, z_{2}, \ldots, z_{n} \in \mathbb{C}$ be some dth roots of unity. If $\operatorname{gcd}(d, n !)=1$, then $z_{1}+z_{2}+\cdots+z_{n} \neq 0$.

Proof. Let $d=q_{1}^{e_{1}} q_{2}^{e_{2}} \cdots q_{s}^{e_{s}}$ be the prime factorization of $d$, where $q_{1}, q_{2}, \ldots, q_{s}$ are distinct prime numbers and $e_{1}, e_{2}, \ldots, e_{s} \geq 1$. Since $\operatorname{gcd}(d, n !)=1$, it follows that $q_{1}>n, q_{2}>n, \cdots, q_{s}>n$. We will prove this lemma by induction on $s$.

First, we consider the case $s=1$. Suppose, for contradiction, that $z_{1}+z_{2}+\cdots+z_{n}=0$. Let $\omega=e^{2 \pi i / d}$. Without loss of generality, we can assume that $z_{1}=\omega^{b_{1}}, z_{2}=\omega^{b_{2}}, \ldots, z_{n}=\omega^{b_{n}}$ with $0=b_{1} \leq b_{2} \leq \cdots \leq b_{n} \leq d-1$. Then

$$
\left(b_{2}-b_{1}\right)+\left(b_{3}-b_{2}\right)+\cdots+\left(b_{n}-b_{n-1}\right)+\left(b_{1}+d-b_{n}\right)=d \text {. }
$$

Since $q_{1}>n$, we have $q_{1}^{e_{1}}>n q_{1}^{e_{1}-1}$, so that $d>n(d-\varphi(d))$, where $\varphi(d)=q_{1}^{e_{1}}-q_{1}^{e_{1}-1}$ is Euler's totient function. Hence, one of $b_{2}-b_{1}, b_{3}-b_{2}, \ldots, b_{n}-b_{n-1}$ and $b_{1}+d-b_{n}=d-b_{n}$ is greater than $d-\varphi(d)$.

Suppose $d-b_{n}>d-\varphi(d)$. Then $b_{n}<\varphi(d)$. Since $z_{1}+z_{2}+\cdots+z_{n}=$ $\omega^{b_{1}}+\omega^{b_{2}}+\cdots+\omega^{b_{n}}=0$, it follows that $\omega$ is a root of the polynomial $x^{b_{1}}+x^{b_{2}}+\cdots+x^{b_{n}}$, which is of degree $b_{n}<\varphi(d)$. But this contradicts the well-known fact that the minimal polynomial of $\omega$ over $\mathbb{Q}$ has degree $\varphi(d)$.

Suppose $b_{i}-b_{i-1}>d-\varphi(d)$ for some $2 \leq i \leq n$. Since $z_{1}+z_{2}+$ $\cdots+z_{n}=0$, it follows that

$$
\frac{1}{z_{i}}\left(z_{1}+z_{2}+\cdots+z_{n}\right)=\frac{1}{z_{i}}\left(z_{i}+z_{i+1}+\cdots+z_{n}+z_{1}+\cdots+z_{i-1}\right)
$$




$$
\begin{aligned}
= & 1+\omega^{b_{i+1}-b i}+\cdots+\omega^{b_{n}-b_{i}}+\omega^{b_{1}+d-b_{i}}+\cdots \\
& +\omega^{b_{i-1}+d-b_{i}}=0 .
\end{aligned}
$$

Hence, $\omega$ is a root of the polynomial $1+x^{b_{i+1}-b i}+\cdots+x^{b_{n}-b_{i}}+x^{d-b_{i}}+$ $\cdots+x^{b_{i-1}+d-b_{i}}$, which is of degree $d-\left(b_{i}-b_{i-1}\right)<d-(d-\varphi(d))=\varphi(d)$. But this also contradicts the fact that the minimal polynomial of $\omega$ over $\mathbb{Q}$ has degree $\varphi(d)$. So we have proved that $z_{1}+z_{2}+\cdots+z_{n} \neq 0$ for the case $s=1$.

Now we consider the case $s \geq 2$. Suppose, for contradiction, that $z_{1}+z_{2}+\cdots+z_{n}=0$. Let $d_{1}=q_{1}^{e_{1}}$ and $d_{2}=q_{2}^{e_{2}} \cdots q_{s}^{e_{s}}$. Then $d=d_{1} d_{2}$ and $\operatorname{gcd}\left(d_{1}, d_{2}\right)=1$. Let $\omega=e^{2 \pi i / d_{1}}, \varepsilon=e^{2 \pi i / d_{2}}$ and $\eta=e^{2 \pi i / d}$. Then it is well known that $\mathbb{Q}(\eta)=\mathbb{Q}(\omega)(\varepsilon)=\mathbb{Q}(\varepsilon)(\omega)$. Hence, without loss of generality, we can assume that $z_{1}=\omega^{b_{1}} \varepsilon^{c_{1}}, z_{2}=$ $\omega^{b_{2}} \varepsilon^{c_{2}}, \ldots, z_{n}=\omega^{b_{n}} \varepsilon^{c_{n}}$ with $0=b_{1} \leq b_{2} \leq \cdots \leq b_{n} \leq d_{1}-1$ and $0=c_{1} \leq c_{2} \leq \cdots \leq c_{n} \leq d_{2}-1$. Then

$$
\left(b_{2}-b_{1}\right)+\left(b_{3}-b_{2}\right)+\cdots+\left(b_{n}-b_{n-1}\right)+\left(b_{1}+d_{1}-b_{n}\right)=d_{1} .
$$

By the proof of case $s=1$, it is easy to see that we can assume $d_{1}-b_{n} \geq$ $\max \left\{b_{i}-b_{i-1} \mid i=2, \ldots, n\right\}$. Since $q_{1}>n$, we have $q_{1}^{e_{1}}>n q_{1}^{e_{1}-1}$, so that $d_{1}>n\left(d_{1}-\varphi\left(d_{1}\right)\right)$, which implies $d_{1}-b_{n}>d_{1}-\varphi\left(d_{1}\right)$, that is, $b_{n}<\varphi\left(d_{1}\right)$.

Let $j=\min \left\{i=1, \ldots, n \mid b_{i}=b_{n}\right\} \geq 1$. Then $\varepsilon^{c_{j}}, \varepsilon^{c_{j+1}}, \ldots, \varepsilon^{c_{n}}$ are some $d_{2}$ th roots of unity. Since $\operatorname{gcd}(d, n !)=1$, it follows that $\operatorname{gcd}\left(d_{2},(n-j+1) !\right)=1$. Thus, by the induction hypothesis, it is easy to see that $\varepsilon^{c_{j}}+\varepsilon^{c_{j+1}}+\cdots+\varepsilon^{c_{n}} \neq 0$. Therefore, $\omega$ is a root of the polynomial $\varepsilon^{c_{1}} x^{b_{1}}+\varepsilon^{c_{2}} x^{b_{2}}+\cdots+\varepsilon^{c_{j-1}} x^{b_{j-1}}+\left(\varepsilon^{c_{j}}+\varepsilon^{c_{j+1}}+\right.$ $\left.\cdots+\varepsilon^{c_{n}}\right) x^{b_{n}} \in \mathbb{Q}(\varepsilon)[x]$, which is of degree $b_{n}<\varphi\left(d_{1}\right)$. However, since $\varphi(d)=\varphi\left(d_{1}\right) \varphi\left(d_{2}\right)$ and

$\varphi(d)=[\mathbb{Q}(\eta): \mathbb{Q}]=[\mathbb{Q}(\varepsilon)(\omega): \mathbb{Q}(\varepsilon)][\mathbb{Q}(\varepsilon): \mathbb{Q}]=[\mathbb{Q}(\varepsilon)(\omega): \mathbb{Q}(\varepsilon)] \varphi\left(d_{2}\right)$,

it follows that $[\mathbb{Q}(\varepsilon)(\omega): \mathbb{Q}(\varepsilon)]=\varphi\left(d_{1}\right)$, so that the minimal polynomial of $\omega$ over $\mathbb{Q}(\varepsilon)$ has degree $\varphi\left(d_{1}\right)$. So we have a contradiction, and therefore, $z_{1}+z_{2}+\cdots+z_{n} \neq 0$.

Proof of Theorem 2.4. Suppose, for contradiction, that $p_{A}(n)$ is not a regular sequence in $S$. Then the polynomial system associated to $p_{A}(n)$ has a nonzero solution $\left(z_{1}, z_{2}, \ldots, z_{n}\right) \in \mathbb{C}^{n}$. Since the polynomials $p_{A}(n)$ are homogeneous and symmetric, without the loss 
of generality, we can assume that $z_{1}=1$ and that $z_{1}^{d}, z_{2}^{d}, \ldots, z_{t}^{d}$ are all the distinct values among $z_{1}^{d}, z_{2}^{d}, \ldots, z_{n}^{d}$. Note that $1 \leq t \leq n$.

If $t=1$ and $d=1$, then $z_{1}=z_{2}=\cdots=z_{n}=1$. Hence, $z_{1}^{a}+z_{2}^{a}+\cdots+z_{n}^{a}=n \neq 0$, which contradicts $\left(z_{1}, z_{2}, \ldots, z_{n}\right)$ being a root of $p_{a}(n)$.

If $t=1$ and $d \geq 2$, then $z_{1}^{d}=z_{2}^{d}=\cdots=z_{n}^{d}=1$. Hence, $z_{1}^{a}, z_{2}^{a}, \ldots, z_{n}^{a}$ are some $d$ th roots of unity. By Lemmas 2.5 and 2.6, we have that $z_{1}^{a}+z_{2}^{a}+\cdots+z_{n}^{a} \neq 0$, which also contradicts $\left(z_{1}, z_{2}, \ldots, z_{n}\right)$ being a root of $p_{a}(n)$.

If $t \geq 2$, then, for every $1 \leq i \leq t$, we set

$$
A_{i}=\left\{j=1,2, \ldots, n \mid z_{j}^{d}=z_{i}^{d}\right\} \quad \text { and } \quad w_{i}=\sum_{j \in A_{i}} z_{j}^{a} .
$$

Since $\left(z_{1}, z_{2}, \ldots, z_{n}\right)$ is a solution of the polynomial system associated to $p_{A}(n)$, we have the following system of equations:

$$
\left\{\begin{array}{l}
w_{1}+w_{2}+\cdots+w_{t}=0 \\
w_{1} z_{1}^{d}+w_{2} z_{2}^{d}+\cdots+w_{t} z_{t}^{d}=0 \\
\cdots \cdots \cdots+\cdots \\
w_{1} z_{1}^{(n-1) d}+w_{2} z_{2}^{(n-1) d}+\cdots+w_{t} z_{t}^{(n-1) d}=0 .
\end{array}\right.
$$

Rewriting the first $t$ equations in the matrix form, we get

$$
\left(\begin{array}{cccc}
1 & 1 & \cdots & 1 \\
z_{1}^{d} & z_{2}^{d} & \cdots & z_{t}^{d} \\
\vdots & \vdots & & \vdots \\
z_{1}^{(t-1) d} & z_{2}^{(t-1) d} & \cdots & z_{t}^{(t-1) d}
\end{array}\right)\left(\begin{array}{c}
w_{1} \\
w_{2} \\
\vdots \\
w_{t}
\end{array}\right)=0 .
$$

By Lemmas 2.5 and 2.6, it is easy to see that $w_{1} \neq 0$, so that $\left(w_{1}, w_{2}, \ldots, w_{t}\right)$ is a nonzero vector. Thus, we have that

$$
\left|\begin{array}{cccc}
1 & 1 & \cdots & 1 \\
z_{1}^{d} & z_{2}^{d} & \cdots & z_{t}^{d} \\
\vdots & \vdots & & \vdots \\
z_{1}^{(t-1) d} & z_{2}^{(t-1) d} & \cdots & z_{t}^{(t-1) d}
\end{array}\right|=0
$$

which implies

$$
\prod_{1 \leq i<j \leq t}\left(z_{j}^{d}-z_{i}^{d}\right)=0
$$


This contradicts the assumption that $z_{1}^{d}, z_{2}^{d}, \ldots, z_{t}^{d}$ are all distinct. Therefore, $p_{A}(n)$ is a regular sequence in $S$.

Remark 2.7. In [2], Conca, et al., verified some special cases of Conjecture 1.1. Now Theorem 2.4 proves some new cases of this conjecture. For example, if $A=\{1,8,15\}$ or $A=\{2,7,12\}$, then $p_{A}(3)$ is a regular sequence in $S=\mathbb{C}\left[x_{1}, x_{2}, x_{3}\right]$. However, Conjecture 1.1 still remains mysterious. For example, if $A=\{2,5,9\}$, then it is hard to see if $p_{A}(3)$ is a regular sequence. Also, when $A=\{1,6, m\}$, the proof for $p_{A}(3)$ being a regular sequence took almost eight pages in [2], so one might wonder if there is an easier way to prove it.

3. Regular sequences close to $x_{1}^{a_{1}}, x_{2}^{a_{2}}, \ldots, x_{n}^{a_{n}}$. Let $S=k\left[x_{1}, x_{2}\right.$, $\left.\ldots, x_{n}\right]$ be the polynomial ring in $n$ variables over an infinite field $k$. Let $f_{1}, f_{2}, \ldots, f_{n}$ be a sequence of homogeneous polynomials of degrees $1 \leq a_{1} \leq a_{2} \leq \cdots \leq a_{n}$ in $S$. Then it is well-known that, generically, $f_{1}, f_{2}, \ldots, f_{n}$ is a regular sequence in $S$. In the next proposition, we will give this basic fact an elementary proof, which will also be useful when proving Theorem 3.5.

Proposition 3.1. Let $S=k\left[x_{1}, x_{2}, \ldots, x_{n}\right]$ with $k$ being any field. Given $1 \leq a_{1} \leq a_{2} \leq \cdots \leq a_{n}$, let $W=S_{a_{1}} \oplus S_{a_{2}} \oplus \cdots \oplus S_{a_{n}}$ be the affine $k$-space of dimension $m$, where $m=\sum_{i=1}^{n}\left(\begin{array}{c}n+a_{i}-1 \\ a_{i}\end{array}\right)$. Let $\Sigma=\left\{\left(f_{1}, f_{2}, \ldots, f_{n}\right) \in W \mid f_{1}, f_{2}, \ldots, f_{n}\right.$ is a regular sequence in $\left.S\right\}$.

Then $\Sigma$ is a non-empty Zariski-open subset of $W$. Furthermore, if $k$ is an infinite field, then $\Sigma$ is a dense open subset of $W$, and then, generically, a sequence of homogeneous polynomials $f_{1}, f_{2}, \ldots, f_{n}$ of degrees $a_{1}, a_{2}, \ldots, a_{n}$ is a regular sequence in $S$.

Proof. For any $d \geq 1$, the set of all monomials in $S_{d}$ ordered in the lexicographic order form a basis of the $k$-vector space $S_{d}$. And $S_{0}=k$ has basis $\{1\}$. These bases give rise to a basis of the $k$-vector space $W=S_{a_{1}} \oplus S_{a_{2}} \oplus \cdots \oplus S_{a_{n}}$. We call these bases the monomial bases of these $k$-vector spaces. So, under the monomial basis, $W$ is isomorphic to $k^{m}$.

Let $N=\sum_{i=1}^{n}\left(a_{i}-1\right)$. Then the $n$-tuple $\left(f_{1}, f_{2}, \ldots, f_{n}\right)$ is in $\Sigma$ if and only if for the ideal $\left(f_{1}, f_{2}, \ldots, f_{n}\right) \subset S$, we have that 
$\left(f_{1}, f_{2}, \ldots, f_{n}\right)_{N}=S_{N}$, or, equivalently, given any $h \in S_{N}$, there exist $g_{1} \in S_{N-a_{1}}, g_{2} \in S_{N-a_{2}}, \ldots, g_{n} \in S_{N-a_{n}}$ such that

$$
f_{1} g_{1}+f_{2} g_{2}+\cdots+f_{n} g_{n}=h .
$$

Under the monomial basis, let $\lambda_{1}, \lambda_{2}, \ldots, \lambda_{m}$ be the coordinates of the $n$-tuple $\left(f_{1}, f_{2}, \ldots, f_{n}\right)$ in $W$, let $\mu_{1}, \mu_{2}, \ldots, \mu_{p}$ be the coordinates of $h$ in $S_{N}$ where $p=\left(\begin{array}{c}n+N-1 \\ N\end{array}\right)$, and let $z_{1}, z_{2}, \ldots, z_{q}$ be the coordinates of the $n$-tuple $\left(g_{1}, g_{2}, \ldots, g_{n}\right)$ in $S_{N-a_{1}} \oplus S_{N-a_{2}} \oplus \cdots \oplus S_{N-a_{n}}$ where $q=\sum_{i=1}^{n}\left(\begin{array}{c}n+N-a_{i}-1 \\ N-a_{i}\end{array}\right)$. Then equation (3.1) is equivalent to the following matrix equation:

$$
A\left(\begin{array}{c}
z_{1} \\
z_{2} \\
\vdots \\
z_{q}
\end{array}\right)=\left(\begin{array}{c}
\mu_{1} \\
\mu_{2} \\
\vdots \\
\mu_{p}
\end{array}\right),
$$

where the $p \times q$ matrix $A$ is determined by equation (3.1) and the entries of $A$ are either some $\lambda_{i}$ or zero. Thus, $f_{1}, f_{2}, \ldots, f_{n}$ is a regular sequence if and only if, given any vector $\left(\mu_{1}, \mu_{2}, \ldots, \mu_{p}\right)^{T}$, there exists a vector $\left(z_{1}, z_{2}, \ldots, z_{q}\right)^{T}$ satisfying equation $(3.2)$, or equivalently, $\operatorname{rank}(A)=p$. So, the $n$-tuple $\left(f_{1}, f_{2}, \ldots, f_{n}\right)$ is in $\Sigma$ if and only if the $m$-tuple $\left(\lambda_{1}, \lambda_{2}, \ldots, \lambda_{m}\right)$ is in $k^{m}-V\left(I_{p}(A)\right)$. Since $k^{m}-V\left(I_{p}(A)\right)$ is a Zariskiopen subset of $k^{m}$, it follows that $\Sigma$ is a Zariski-open subset of $W$. Note that $x_{1}^{a_{1}}, x_{2}^{a_{2}}, \ldots, x_{n}^{a_{n}}$ is a regular sequence in $S$. Therefore, $\Sigma$ is a non-empty Zariski-open subset of $W$.

If the field $k$ is infinite, then the affine space $W$ is irreducible, so that the non-empty Zariski-open subset $\Sigma$ is dense in $W$, which implies that, generically, a sequence of homogeneous polynomials $f_{1}, f_{2}, \ldots, f_{n}$ of degrees $a_{1}, a_{2}, \ldots, a_{n}$ is a regular sequence in $S$.

To illustrate equation (3.2) in the above proof of Proposition 3.1, we have the following example.

Example 3.2. Let $S=k\left[x_{1}, x_{2}\right]$ and $a_{1}=a_{2}=2$. Then the numbers defined in the above proof are $m=6, N=3$ and $p=q=4$. Let

$$
\begin{array}{ll}
f_{1}=\lambda_{1} x_{1}^{2}+\lambda_{2} x_{1} x_{2}+\lambda_{3} x_{2}^{2}, & g_{1}=z_{1} x_{1}+z_{2} x_{2}, \\
f_{2}=\lambda_{4} x_{1}^{2}+\lambda_{5} x_{1} x_{2}+\lambda_{6} x_{2}^{2}, & g_{2}=z_{3} x_{1}+z_{4} x_{2},
\end{array}
$$




$$
h=\mu_{1} x_{1}^{3}+\mu_{2} x_{1}^{2} x_{2}+\mu_{3} x_{1} x_{2}^{2}+\mu_{4} x_{2}^{3} .
$$

Then $f_{1} g_{1}+f_{2} g_{2}=h$ is equivalent to the following matrix equation:

$$
A\left(\begin{array}{c}
z_{1} \\
z_{2} \\
z_{3} \\
z_{4}
\end{array}\right)=\left(\begin{array}{cccc}
\lambda_{1} & 0 & \lambda_{4} & 0 \\
\lambda_{2} & \lambda_{1} & \lambda_{5} & \lambda_{4} \\
\lambda_{3} & \lambda_{2} & \lambda_{6} & \lambda_{5} \\
0 & \lambda_{3} & 0 & \lambda_{6}
\end{array}\right)\left(\begin{array}{c}
z_{1} \\
z_{2} \\
z_{3} \\
z_{4}
\end{array}\right)=\left(\begin{array}{c}
\mu_{1} \\
\mu_{2} \\
\mu_{3} \\
\mu_{4}
\end{array}\right) .
$$

Since $z_{1}$ in $g_{1}$ can only be multiplied by $f_{1}$, it follows that only the coefficients $\lambda_{1}, \lambda_{2}, \lambda_{3}$ of $f_{1}$ can appear in the first column of the matrix $A$ and each coefficient of $f_{1}$ appears only once in the first column. The other columns of $A$ have similar properties. Also, one can see that $f_{1}, f_{2}$ is a regular sequence in $S$ if and only if $\operatorname{det}(A) \neq 0$.

Remark 3.3. Illustrated by the above example, we have that, in general, the matrix $A$ in the proof of Proposition 3.1 has the following property: for any column of $A$, there exists some $f_{i}$ such that the entries of the column are either the coefficients of $f_{i}$ or zero, and each coefficient of $f_{i}$ appears only once in that column. This observation about the matrix $A$ will be used in the proof of Theorem 3.5, which is the main result of this section.

If $k=\mathbb{C}$, then by [3, Theorem 2.3, Chapter 3], we see that $f_{1}, f_{2}, \ldots, f_{n}$ is a regular sequence in $S$ if and only if the resultant $\operatorname{Res}\left(f_{1}, f_{2}, \ldots, f_{n}\right)$ is nonzero. If $n=2$ or $a_{1}=a_{2}=\cdots=a_{n}=1$, then $\operatorname{Res}\left(f_{1}, f_{2}, \ldots, f_{n}\right)=\operatorname{det}(A)$. For other cases, the matrix $A$ is not even a square matrix. And, although we have that $V\left(I_{p}(A)\right)=$ $V\left(\operatorname{Res}\left(f_{1}, f_{2}, \ldots, f_{n}\right)\right)$, it is not clear if $\operatorname{Res}\left(f_{1}, f_{2}, \ldots, f_{n}\right)$ can always be expressed as a single determinant.

In order to state Theorem 3.5, we need the following definition. In the rest of this section, we will assume $k=\mathbb{C}$.

Definition 3.4. Let $S=\mathbb{C}\left[x_{1}, x_{2}, \ldots, x_{n}\right]$ and $f, g$ be two homogeneous polynomials in $S$ of degree $d$. Suppose

$$
\begin{aligned}
& f=\lambda_{1} x_{1}^{d}+\lambda_{2} x_{1}^{d-1} x_{2}+\cdots+\lambda_{r} x_{n}^{d}, \\
& g=\nu_{1} x_{1}^{d}+\nu_{2} x_{1}^{d-1} x_{2}+\cdots+\nu_{r} x_{n}^{d},
\end{aligned}
$$


where $r=\left(\begin{array}{c}n+d-1 \\ d\end{array}\right)$. Then we define

$$
\mathrm{d}(f, g)=\left|\lambda_{1}-\nu_{1}\right|+\left|\lambda_{2}-\nu_{2}\right|+\cdots+\left|\lambda_{r}-\nu_{r}\right|,
$$

and we call it the distance between $f$ and $g$.

Given $1 \leq a_{1} \leq a_{2} \leq \cdots \leq a_{n}$, let $W=S_{a_{1}} \oplus S_{a_{2}} \oplus \cdots \oplus S_{a_{n}}$. For any two $n$-tuples $\left(f_{1}, f_{2}, \ldots, f_{n}\right)$ and $\left(g_{1}, g_{2}, \ldots, g_{n}\right)$ in $W$, we can define the distance between them as follows:

$\mathrm{d}\left(\left(f_{1}, f_{2}, \ldots, f_{n}\right),\left(g_{1}, g_{2}, \ldots, g_{n}\right)\right)=\mathrm{d}\left(f_{1}, g_{1}\right)+\mathrm{d}\left(f_{2}, g_{2}\right)+\cdots+\mathrm{d}\left(f_{n}, g_{n}\right)$.

This definition makes $W$ into a metric space, whose induced topology is larger than the Zariski topology on $W$. So, by Proposition 3.1, the set $\Sigma$ is a non-empty open subset in the metric space $W$. Therefore, given any regular sequence $f_{1}, f_{2}, \ldots, f_{n}$ of homogeneous polynomials with degrees $a_{1}, a_{2}, \ldots, a_{n}$ in $S=\mathbb{C}\left[x_{1}, x_{2}, \ldots, x_{n}\right]$, there exists $\varepsilon>0$ such that, for any homogeneous polynomials $g_{1}, g_{2}, \ldots, g_{n} \in S$ of degrees $a_{1}, a_{2}, \ldots, a_{n}$, if $\mathrm{d}\left(g_{1}, f_{1}\right)<\varepsilon, \mathrm{d}\left(g_{2}, f_{2}\right)<\varepsilon, \ldots, \mathrm{d}\left(g_{n}, f_{n}\right)<\varepsilon$, then $g_{1}, g_{2}, \ldots, g_{n}$ is also a regular sequence in $S$.

The question is: if $f_{1}=x_{1}^{a_{1}}, f_{2}=x_{2}^{a_{2}}, \ldots, f_{n}=x_{n}^{a_{n}}$, what is the maximal value for $\varepsilon$ ? The answer is $\varepsilon=1$, as is shown in the following theorem.

Theorem 3.5. Let $f_{1}, f_{2}, \ldots, f_{n}$ be a sequence of homogeneous polynomials of degrees $1 \leq a_{1} \leq a_{2} \leq \cdots \leq a_{n}$ in $S=\mathbb{C}\left[x_{1}, x_{2}, \ldots, x_{n}\right]$. If $\mathrm{d}\left(f_{i}, x_{i}^{a_{i}}\right)<1$ for $i=1,2, \ldots, n$, then $f_{1}, f_{2}, \ldots, f_{n}$ is a regular sequence in $S$.

To prove this theorem, we need the following lemma.

Lemma 3.6. Let $A=\left(a_{i j}\right)_{n \times n} \in M_{n}(\mathbb{C})$. If, for every $j=1,2, \ldots, n$, we have

$$
\left|a_{j j}\right|>\left|a_{1 j}\right|+\cdots+\left|a_{j-1, j}\right|+\left|a_{j+1, j}\right|+\cdots+\left|a_{n j}\right|,
$$

then $\operatorname{det}(A) \neq 0$.

Proof. Suppose, for contradiction, that $\operatorname{det}(A)=0$. Let $\alpha_{i}=$ $\left(a_{i 1}, a_{i 2}, \ldots, a_{i n}\right)$ for $i=1,2, \ldots, n$. Then $\alpha_{1}, \alpha_{2}, \ldots, \alpha_{n}$ are linearly 
dependent. Hence, there exist $l_{1}, l_{2}, \ldots, l_{n} \in \mathbb{C}$, which are not all zero, such that

$$
l_{1} \alpha_{1}+l_{2} \alpha_{2}+\cdots+l_{n} \alpha_{n}=0 .
$$

Let $\left|l_{j}\right|=\max \left\{\left|l_{i}\right| \mid i=1,2, \ldots, n\right\}>0$. Then

$$
\alpha_{j}=-\frac{l_{1}}{l_{j}} \alpha_{1}-\cdots-\frac{l_{j-1}}{l_{j}} \alpha_{j-1}-\frac{l_{j+1}}{l_{j}} \alpha_{j+1}-\frac{l_{n}}{l_{j}} \alpha_{n} .
$$

Hence,

$$
a_{j j}=-\frac{l_{1}}{l_{j}} a_{1 j}-\cdots-\frac{l_{j-1}}{l_{j}} a_{j-1, j}-\frac{l_{j+1}}{l_{j}} a_{j+1, j}-\frac{l_{n}}{l_{j}} a_{n j} .
$$

Thus,

$$
\begin{aligned}
\left|a_{j j}\right| \leq & \left|\frac{l_{1}}{l_{j}}\right|\left|a_{1 j}\right|+\cdots+\left|\frac{l_{j-1}}{l_{j}}\right|\left|a_{j-1, j}\right| \\
& +\left|\frac{l_{j+1}}{l_{j}}\right|\left|a_{j+1, j}\right|+\left|\frac{l_{n}}{l_{j}}\right|\left|a_{n j}\right| \\
\leq & \left|a_{1 j}\right|+\cdots+\left|a_{j-1, j}\right|+\left|a_{j+1, j}\right|+\left|a_{n j}\right|,
\end{aligned}
$$

which contradicts the assumption. Therefore, $\operatorname{det}(A) \neq 0$.

Proof of Theorem 3.5. For every $i=1,2, \ldots, n$, let $\nu_{i}$ be the coefficient of $x_{i}^{a_{i}}$ in $f_{i}$ and $c_{i}$ the sum of the absolute value of the other coefficients in $f_{i}$. Since $\mathrm{d}\left(f_{i}, x_{i}^{a_{i}}\right)<1$, it follows that $c_{i}+\left|\nu_{i}-1\right|<1$, which implies that $c_{i}<1-\left|\nu_{i}-1\right| \leq\left|1+\left(\nu_{i}-1\right)\right|=\left|\nu_{i}\right|$.

Let $A$ be the $p \times q$ matrix defined in the proof of Proposition 3.1. Since $x_{1}^{a_{1}}, x_{2}^{a_{2}}, \ldots, x_{n}^{a_{n}}$ is a regular sequence in $S$, it follows that there is a $p \times p$ submatrix $B$ of $A$, which is invertible when evaluated at the $n$-tuple $\left(x_{1}^{a_{1}}, x_{2}^{a_{2}}, \ldots, x_{n}^{a_{n}}\right)$. Let $B\left(x_{1}^{a_{1}}, x_{2}^{a_{2}}, \ldots, x_{n}^{a_{n}}\right)$ denote the matrix $B$ evaluated at $\left(x_{1}^{a_{1}}, x_{2}^{a_{2}}, \ldots, x_{n}^{a_{n}}\right)$. By Remark 3.3, it is easy to see that the column vectors of $B\left(x_{1}^{a_{1}}, x_{2}^{a_{2}}, \ldots, x_{n}^{a_{n}}\right)$ are of the form $(0, \ldots, 0,1,0, \ldots, 0)^{T}$. Since $B\left(x_{1}^{a_{1}}, x_{2}^{a_{2}}, \ldots, x_{n}^{a_{n}}\right)$ is invertible, by rearranging the column vectors of $B$, we can assume that $B\left(x_{1}^{a_{1}}, x_{2}^{a_{2}}, \ldots, x_{n}^{a_{n}}\right)$ is the identity matrix.

Let $B\left(f_{1}, f_{2}, \ldots, f_{n}\right)$ be the matrix $B$ evaluated at the $n$-tuple $\left(f_{1}, f_{2}, \ldots, f_{n}\right)$. For every $i=1,2, \ldots, p$, let $\eta_{i}$ be the $i$ th column vector of $B\left(f_{1}, f_{2}, \ldots, f_{n}\right)$. Then, by Remark 3.3 , it is easy to see that the $i$ th entry in $\eta_{i}$ is $\nu_{j}$ for some $1 \leq j \leq n$, all the other 
coefficients of $f_{j}$ appear as the entries of $\eta_{i}$ exactly once, and the remaining entries of $\eta_{i}$ are zeros. Since $c_{j}<\left|\nu_{j}\right|$, it follows that the matrix $B\left(f_{1}, f_{2}, \ldots, f_{n}\right)$ satisfies the assumptions of Lemma 3.6, and then $\operatorname{det}\left(B\left(f_{1}, f_{2}, \ldots, f_{n}\right)\right) \neq 0$. So the matrix $A$ evaluated at the $n$ tuple $\left(f_{1}, f_{2}, \ldots, f_{n}\right)$ has rank $p$. Therefore, $f_{1}, f_{2}, \ldots, f_{n}$ is a regular sequence in $S$.

Remark 3.7. It is easy to see that in $S=\mathbb{C}\left[x_{1}, x_{2}\right]$, if $\mathrm{d}\left(f_{1}, x_{1}^{a_{1}}\right)=1$ and $\mathrm{d}\left(f_{2}, x_{2}^{a_{2}}\right)=1$, then $f_{1}, f_{2}$ may not be a regular sequence in $S$. For example, $f_{1}=x^{2}+x y, f_{2}=x y^{2}+y^{3}$ is not a regular sequence in $S$.

The regular sequences obtained in Theorem 3.5 are very different from the regular sequences obtained in Theorem 2.4. It would be interesting to know if the EGH conjecture holds for these two classes of regular sequences. Besides, the above two classes of regular sequences are in $S=\mathbb{C}\left[x_{1}, x_{2}, \ldots, x_{n}\right]$. If $S=\mathbb{Z} / 2 \mathbb{Z}\left[x_{1}, x_{2}, \ldots, x_{n}\right]$, then the sequence in Theorem 2.4 is not a regular sequence because the polynomial system has a non-zero solution $(1,1,0, \ldots, 0)$. It would be interesting to find a new class of regular sequences in $S=\mathbb{Z} / 2 \mathbb{Z}\left[x_{1}, x_{2}, \ldots, x_{n}\right]$.

We end this paper with the following proposition. We put it in this paper because its proof is similar to the proof of Proposition 3.1.

Proposition 3.8. Let $S=\mathbb{C}\left[x_{1}, x_{2}, \ldots, x_{n}\right]$. Let $f_{1}, f_{2}, \ldots, f_{t}$ be $\mathbb{C}$ linearly independent polynomials in $S_{i}$ with $i \geq 1$. Then there exists $\varepsilon>0$ such that, for any homogeneous polynomials $g_{1}, g_{2}, \ldots, g_{t}$ in $S_{i}$ satisfying $\mathrm{d}\left(g_{1}, f_{1}\right)<\varepsilon, \mathrm{d}\left(g_{2}, f_{2}\right)<\varepsilon, \ldots, \mathrm{d}\left(g_{t}, f_{t}\right)<\varepsilon$, we have that $g_{1}, g_{2}, \ldots, g_{t}$ are $\mathbb{C}$-linearly independent and

$$
\operatorname{dim}_{\mathbb{C}}\left(f_{1}, f_{2}, \ldots, f_{t}\right)_{i+1} \leq \operatorname{dim}_{\mathbb{C}}\left(g_{1}, g_{2}, \ldots, g_{t}\right)_{i+1} .
$$

Proof. It is easy to see that $g_{1}, g_{2}, \ldots, g_{t}$ are $\mathbb{C}$-linearly independent when $\varepsilon$ is sufficiently small. Let

$$
\begin{aligned}
\Sigma=\left\{\left(g_{1}, g_{2}, \ldots, g_{t}\right) \in\left(S_{i}\right)^{t} \mid \operatorname{dim}_{\mathbb{C}}\left(g_{1}, g_{2}, \ldots, g_{t}\right)_{i+1}\right. \\
\left.\quad \geq \operatorname{dim}_{\mathbb{C}}\left(f_{1}, f_{2}, \ldots, f_{t}\right)_{i+1}\right\} .
\end{aligned}
$$

Since the $t$-tuple $\left(f_{1}, f_{2}, \ldots, f_{t}\right)$ is in $\Sigma$, it follows that $\Sigma$ is non-empty, so that it suffices to prove that $\sigma$ is an open subset of the affine $\mathbb{C}$-space 
$\left(S_{i}\right)^{t}$. Note that under the monomial basis as defined in the proof of Proposition 3.1, $\left(S_{i}\right)^{t}$ is isomorphic to $\mathbb{C}^{N}$ where $N=t\left(\begin{array}{c}n+i-1 \\ i\end{array}\right)$.

For any $t$-tuple $\left(g_{1}, g_{2}, \ldots, g_{t}\right) \in\left(S_{i}\right)^{t},\left(g_{1}, g_{2}, \ldots, g_{t}\right)_{i+1}$ is the $\mathbb{C}$ vector space spanned by the $n t$ polynomials $x_{1} g_{1}, x_{2} g_{1}, \ldots, x_{n} g_{t}$. Let $B$ be the $n t \times\left(\begin{array}{l}n+i \\ i+1\end{array}\right)$ matrix with each row being the coordinates of some $x_{l} g_{j}$ under the monomial basis of $S_{i+1}$, where $l=1,2, \ldots, n$ and $j=1,2, \ldots, t$. If the coordinates of $t$-tuple $\left(g_{1}, g_{2}, \ldots, g_{t}\right)$ under the monomial basis of $\left(S_{i}\right)^{t}$ is $\left(\lambda_{1}, \lambda_{2}, \ldots, \lambda_{N}\right)$, then the entries of $B$ are either some $\lambda_{i}$ or zero. Let $p=\operatorname{dim}_{\mathbb{C}}\left(f_{1}, f_{2}, \ldots, f_{t}\right)_{i+1}$, then it is easy to see that the $t$-tuple $\left(g_{1}, g_{2}, \ldots, g_{t}\right)$ is in $\Sigma$ if and only if the $N$-tuple $\left(\lambda_{1}, \lambda_{2}, \ldots, \lambda_{N}\right)$ is in $\mathbb{C}^{N}-V\left(I_{p}(B)\right)$. Since $\mathbb{C}^{N}-V\left(I_{p}(B)\right)$ is an open subset of $\mathbb{C}^{N}$, it follows that $\Sigma$ is an open subset of $\left(S_{i}\right)^{t}$.

Acknowledgments. The author would like to thank the referee for his/her valuable comments.

\section{REFERENCES}

1. A. Abedelfatah, On the Eisenbud-Green-Harris conjecture, Proc. Amer. Math. Soc. 143 (2015), 105-115.

2. A. Conca, C. Krattenthaler and J. Watanabe, Regular sequences of symmetric polynomials, Rend. Sem. Mat. Univ. Padova 121 (2009), 179-199.

3. D. Cox, J. Little and D. O'Shea, Using algebraic geometry, Second edition, Grad. Texts Math. 227, Springer-Verlag, New York, 2005.

4. D. Eisenbud, M. Green and J. Harris, Higher Castelnuovo theory, Astérisque 218 (1993), 187-202.

5. T.Y. Lam and K.H. Leung, On vanishing sums of roots of unity, J. Alg. 224 (2000), 91-109.

Department of Mathematics, Nanjing University of Science and TechnolOGY, NANJing, Jiangsu, 210094, P.R.China

Email address: rc429@cornell.edu 